\title{
Stereo MicroPIV measurements in an irrigation nozzle
}

\author{
F. Gökhan Ergin ${ }^{1, *}$, Séverine Tomas ${ }^{2}$, and Claudiu Pătrașcu ${ }^{3}$ \\ ${ }^{1}$ Dantec Dynamics, Tonsbakken 16-18, Skovlunde, Denmark \\ ${ }^{2}$ G-EAU, AgroParisTech, Cirad, IRD, IRSTEA, MontpellierSupAgro, Univ Montpellier, France \\ ${ }^{3}$ Hydraulics Department, Polytechnic University of Bucharest, Romania
}

\begin{abstract}
Irrigation nozzles often feature small serpentine-shaped channels to avoid clogging. Reduced clogging improves the lifetime of the irrigation nozzle, which reduces plastic waste and thereby reduces the impact on the environment. Clogging in micro channels is often suppressed in the presence of threedimensional (3D) flow structures called vortices. In micro scales the initiation of such 3D microstructures is normally suppressed because of the low Reynolds number inherent to micro scale flows. Passive, zig-zag shaped irrigation nozzles have the potential to induce three- dimensional rotating flow structures around sharp corners, which enhance $3 \mathrm{D}$ flows in the channel and thereby reduce clogging. The aim of this study is to identify and characterize such vortices using a Stereoscopic MicroPIV system.
\end{abstract}

\section{Introduction}

Irrigation nozzles are passive devices which rely entirely on the fluid pumping energy. In these devices, the channel geometry is the main design parameter that affects the observed flow field in the channel. Many channel designs have been used in irrigation nozzles as channel topology has a direct effect on pumping energy and clogging, and these designs have an indirect impact on the environment: On one hand, the aim is to maintain a laminar flow in the channel to keep low pumping costs. However, this approach often leads to clogging problems in the irrigation nozzles. On the other hand, clogging reduces the useful lifetime of the irrigation nozzle, which produces more plastic waste over time and this has a negative impact on the environment. So often what is desired is a channel geometry that can achieve low pumping costs and reduced clogging at the same time. The subject of this work is a serpentine, or zig-zag shaped geometry which has the potential to deliver reduced clogging with an acceptable pumping cost.

Clogging is often suppressed in the presence of threedimensional (3D) flow structures called vortices. In micro scales the initiation of such 3D micro flow structures is normally suppressed because of the low Reynolds number inherent to micro scale flows. This is because, flows have a tendency to stay laminar in low Reynolds numbers and mixing is often result of molecular diffusion between the involved species. Passive, zig-zag shaped irrigation nozzles have the potential to induce 3D rotating flow structures around sharp corners, even in low Reynolds numbers. This enhances 3D flows in the channel and thereby has the potential to reduce clogging. The aim of this study is to

\footnotetext{
* Corresponding author: gokhan.ergin@dantecdynamics.com
}

identify and characterize such vortices using a Stereoscopic MicroPIV system.

\section{Experiments}

Experiments are performed on the flow through a serpentine microchannel in order to check the presence of micro scale vortices. The microchannel dimensions are described in [1] and shown here in Fig. 1. The channel depth is $1.15 \mathrm{~mm}$, which is rather large compared to what can be achieved with current manufacturing techniques. In the current study, the results from a single measurement plane (center-plane) is reported.

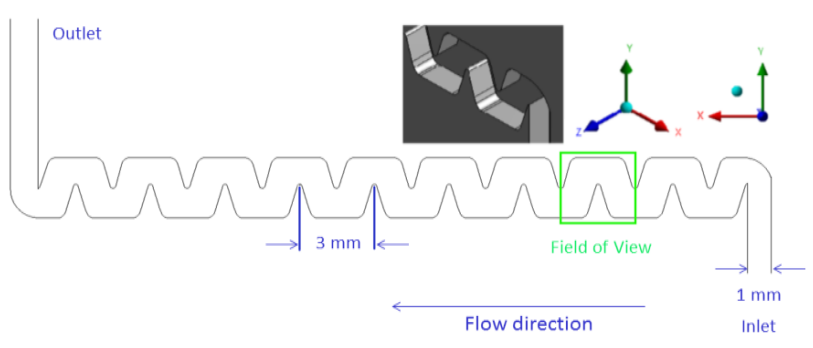

Fig. 1. Serpentine irrigation nozzle from [1]. Measurements are taken in the 3rd baffle.

Stereoscopic MicroPIV technique [2, 3] has received increased attention recently, as it can provide the necessary multiple-component velocity information in the investigated irrigation nozzle with micron scale resolution. Experiments are performed to measure all three velocity components in the center height of the microchannel, around the $3 \mathrm{rd}$ baffle. The experimental setup includes a Leica fluorescence stereomicroscope, 1× Plan Apochromatic common main objective, a custom-built micro image calibration kit (Fig. 2), laser and LED illumination, and a checkerboard calibration 
plate (Fig. 3). The selected microscope and objective configuration can produce a stereoscopic half-angle of $\sim 11^{\circ}$ in air (full angle $22^{\circ}$ ), and this means that the uncertainty of the out-of-plane velocity component, $\mathrm{w}$, is expected as 5 times that of the in-plane velocity components, $\mathrm{u}$ and $\mathrm{v}$ [4].

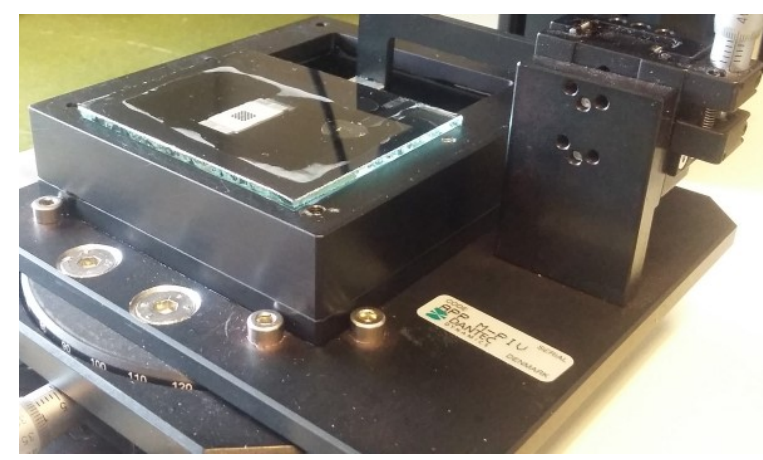

Fig. 2. Stereoscopic image calibration kit for MicroPIV.

Stereoscopic image calibration in a microchannel is simply difficult, if not impossible. In order to achieve an accurate representation of the object space in $3 \mathrm{D}$, a dedicated image calibration kit is used, which consists of a checkerboard calibration target, a calibration pool and a microstage to traverse the target in the calibration pool. The cover glass of the calibration pool and of the microchannel are the same material $(2 \mathrm{~mm}$ thick glass for the current experiment) simulating similar optical conditions during calibration and measurements.

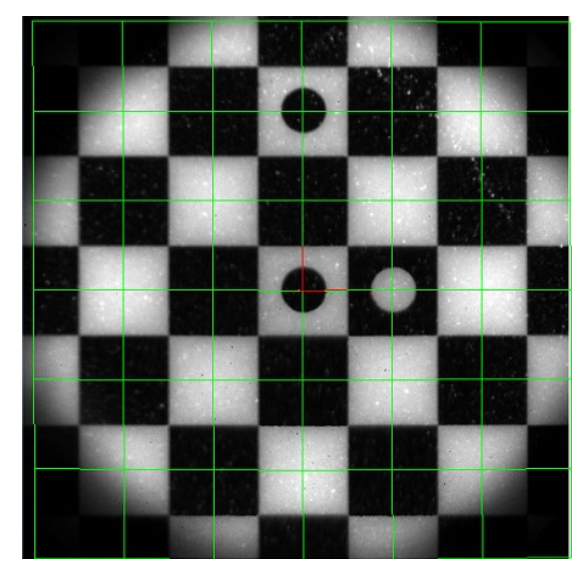

Fig. 3. View of the checkerboard calibration target with the fitted coordinate system (red) and metric calibration grid (green). Each square edge dimension is $500 \mu \mathrm{m}$.

The calibration target features a checkerboard pattern for a $4.5 \mathrm{~mm}$ object size. Carefully placed three circular markers within the squares allow an unambiguous definition of the planar coordinate axes. During calibration, a pulsed mono- chromatic LED device is used for illumination, which proves useful in obtaining calibration images with good contrast. The calibration is performed (i) by imaging the checkerboard pattern in known positions within the depth of field (ii) by computing mapping function for the $3 \mathrm{D}$ space using a 3 rd order polynomial function [5] and (iii) by performing calibration refinement. The depth of field is adjusted by closing the aperture of the zoom optics of the stereomicroscope.

A depth calibration is also performed for accurate positioning of the measurement plane in the microchannel. The depth calibration, stereoscopic calibration and experiments are performed at $5 \times$ total system magnification, which produces a Field of View (FoV) of $3 \mathrm{~mm} \times 3 \mathrm{~mm}$. During depth calibration and measurements the aperture was adjusted to produce a $31 \mu \mathrm{m}$ field depth (important for depth calibration) and $385 \mu \mathrm{m}$ correlation depth (important for experiments). Five calibration images were used at $\mathrm{h}= \pm 200 \mu \mathrm{m}$, $\pm 100 \mu \mathrm{m}$ and $0 \mu \mathrm{m}$ (center height). The average reprojection error for left and right cameras were found as $\sim 1$ pixel as a result of image calibration using a new marker detection algorithm.

A high precision syringe pump with a linear step resolution of $12 \mathrm{~nm}$ is used to deliver the distilled water seeded with 1-20 $\mu \mathrm{m}$-diameter, Rhodamine B fluorescent particles. The syringe pump's flow rate accuracy and reproducibility are given as $\pm 0.35 \%$ and $\pm 0.05 \%$ respectively. The syringe pump can drive two syringes simultaneously and the flow rate is adjustable between $1.6 \mathrm{pl} / \mathrm{min}$ to $300 \mathrm{ml} / \mathrm{min}$ using different syringe sizes. High quality airtight glass syringes are used to generate a smooth flow during the measurements. Two flow rate settings of $24 \mathrm{ml} / \mathrm{min}$ and $48 \mathrm{ml} / \mathrm{min}$ producing Reynolds numbers of 400 and 800 respectively. The results of only $\mathrm{Re}=400$ are presented here for brevity, and the results for $\mathrm{Re}=800$ are presented during the conference. The flow rates are selected so that they can be compared with the 2D2C PIV results obtained in [1].

During measurements, pulsed illumination at $532 \mathrm{~nm}$ from a $60 \mathrm{~mJ} /$ pulse dual-cavity $\mathrm{Nd}$ :YAG laser is delivered to the microscope using a liquid light guide and imaging is performed using two 12-bit FlowSenseEO 4Mpix (2048pix×2048pix) PIV cameras. Planar three component velocity measurements are computed by combining the $2 \mathrm{D} 2 \mathrm{C}$ velocity field information from each camera, and refined image calibration information. Contrast of raw particle images was enhanced by performing a background subtraction using the minimum pixel value found in the ensemble. An ensemble static masking technique is applied to perform vector masking.

\section{Results}

It is often challenging to find the intricate flow details when the complicated flow field is observed using the $2 \mathrm{D}$ view where $\mathrm{u}$ and $\mathrm{v}$ are represented by vectors and $\mathrm{w}$ is represented in colors (Fig. 4). In the $2 \mathrm{D}$ view, a large vortex can be observed on the lee side of each sharp turn. Two such vortices are observed in Fig. 4, one in the bottom left corner and one in the top right corner. These large $3 \mathrm{D}$ vortices are exactly what is required to reduce clogging in irrigation devices. Additionally, the $3 \mathrm{D}$ view of the results reveal smaller and oblique micro vortex systems originating from the corners of the serpentine irrigation nozzle (Fig. 5). These small-scale vortices are only recognizable using only the $3 \mathrm{D}$ view and from a 
particular angle that is parallel to the local maximum velocity. A similar vortex is observed using the $3 \mathrm{D}$ view that originates from the sharp corner close to the left of the FoV in Fig. 4 (3D view for this is not shown here for brevity). In short, one large counter-clockwise vortex (Fig. 4) and one smaller, oblique, clockwise vortex (Fig. 5) are produced at each turn.

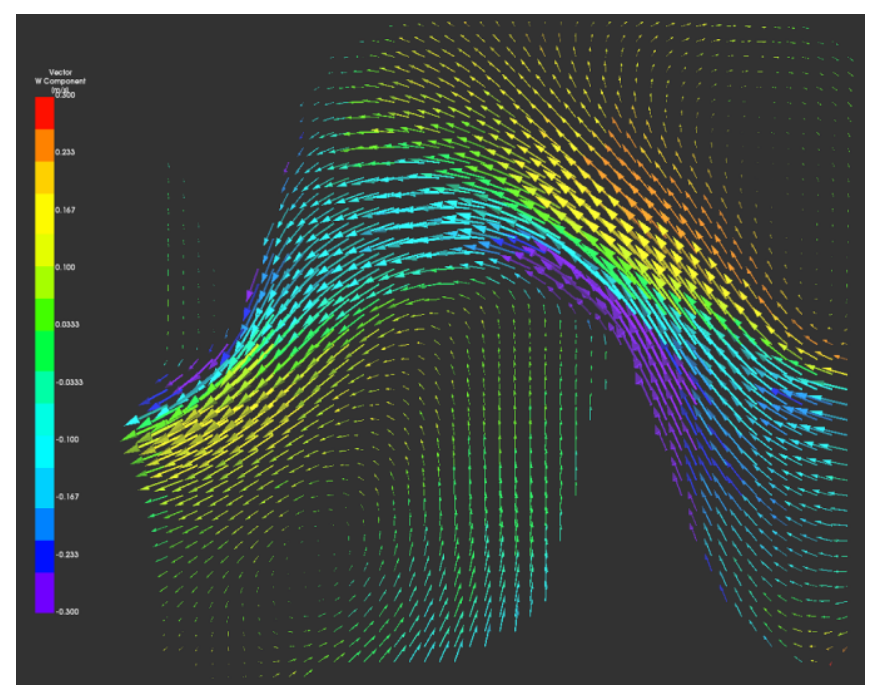

Fig. 4. 2D view of the mean velocity field. (Average of 910 three-component velocity fields). Colors indicate the out-ofplane velocity component $(\mathrm{w}< \pm 0.3 \mathrm{~m} / \mathrm{s})$.

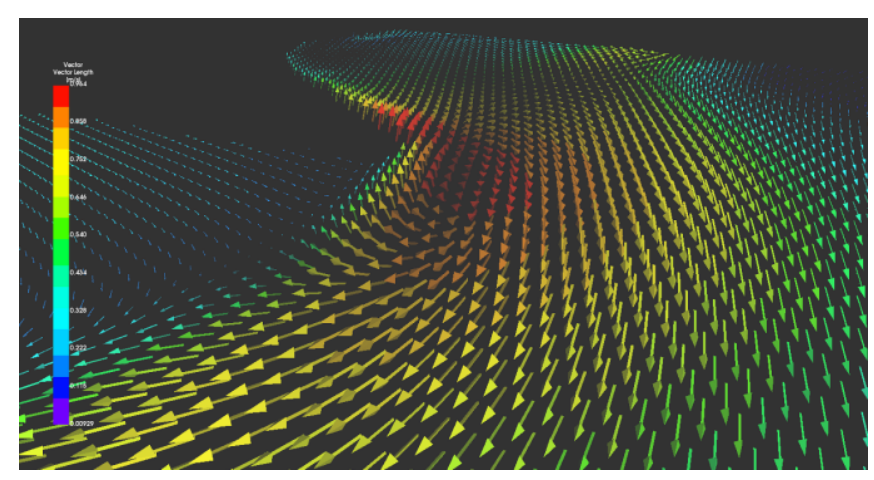

Fig. 5. 3D view of the mean velocity field (as in Fig. 4) showing a large vortex observed originating from to one of the sharp corners of the serpentine irrigation nozzle. Colors indicate the magnitude of the velocity vector $(0<\mathrm{V}<1 \mathrm{~m} / \mathrm{s})$.

\section{Conclusions and Outlook}

It was demonstrated that Stereo MicroPIV is a very suitable technique for performing three-component velocity measurements in microfluidic devices and in particular for mm-sized irrigation nozzles. The following statements can be made for the experiments: First, it was possible to perform stereoscopic image calibration and the depth calibration through the $2 \mathrm{~mm}$-thick glass windows. This is normally achieved with thinner $(<1 \mathrm{~mm}$-thick) windows. Second, it was possible to obtain the flow field in the centre of the channel with $\sim 25 \mu \mathrm{m}$ spatial resolution. Third, it was possible to visualize larger in-plane vortices and smaller oblique vortices originating from the sharp corners of the irrigation nozzle. The latter is achieved using a special
3D viewer. To conclude, two vortices with opposite rotations are produced at each turn: one large counterclockwise vortex (Fig. 4) and one smaller, oblique, clockwise vortex (Fig. 5). Future experiments will be performed at different channel heights and at different flow rates in order to get a complete $3 \mathrm{D}$ mapping of the flow field.

\section{References}

1. Al-Muhammad J, Tomas S, Ait-Mouheb N, Amielh $\mathrm{M}$, Anselmet $\mathrm{F}$ " $\mu \mathrm{PIV}$ characterization of the flow in a milli-labyrinth-channel used in microirrigation" 17th Int. Symp. on Applications of Laser Techniques To Fluid Mechanics. Lisbon, Portugal, 07-10 July, 2014

2. Lindken, R., Westerweel, J., \& Wieneke, B. (2006). Stereoscopic micro particle image velocimetry. Experiments in Fluids, 41(2), 161-171

3. Brede, M., Witte, M., \& Leder, A. (2006) StereoMicro PIV measurements of the three- dimensional separated flow in the wake of a backward facing step. Proc. 13th Int Symp on Applications of Laser Techniques to Fluid Mechanics

4. Prasad, A.K., (2000) Stereoscopic Particle Image Velocimetry. Experiments in Fluids, 29, 103-116

5. Soloff, S.M., Adrian R.J., \& Liu Z.-C. (1997) Distortion compensation for generalized stereoscopic particle image velocimetry. Meas. Sci. Tech. in Fluids, 8, 1441-1454 